\title{
The Equation of the Universe (According to the Theory of Relation)
}

\author{
Russell Bagdoo \\ Saint-Bruno-de-Montarville, Quebec, Canada \\ Email: rbagdoo@gmail.com, rbagdoo@yahoo.ca, mberbag@videotron.ca
}

How to cite this paper: Bagdoo, R. (2019) The Equation of the Universe (According to the Theory of Relation). Journal of Modern Physics, 10, 310-343.

https://doi.org/10.4236/jmp.2019.103022

Received: December 25, 2018

Accepted: March 11, 2019

Published: March 14, 2019

Copyright $\odot 2019$ by author(s) and Scientific Research Publishing Inc. This work is licensed under the Creative Commons Attribution International License (CC BY 4.0).

http://creativecommons.org/licenses/by/4.0/ (c) (i) Open Access

\begin{abstract}
A new equation is found in which the concept of matter-space-time is mathematically connected; gravitation and electromagnetism are also bound by space-time. A mechanism is described showing how velocity, time, distance, matter, and energy are correlated. We are led to ascertain that gravity and electricity are two distinct manifestations of a single underlying process: electro-gravitation. The force of gravitation arises of electromagnetism-inherently much stronger-divided by the cosmological space-time. The radius of space-time belongs to the family of electromagnetic waves: the wavelength is the radius $\left(10^{26} \mathrm{~m}\right)$ of the universe and the period $\left(10^{17} \mathrm{~s}\right)$ is its cosmological age. For the first time, the cosmological time, considered as a real physical object, is integrated into a "cosmological equation" which makes coherent what we know regarding the time (its origin, its flow ...), the matter, and space. It sets up a mathematical model allowing us to interpret dark energy (or cosmological constant) as being both "negative" and "tired" energy. After an introduction with a brief history of unifications and the presentation of two roughly equal ratios arising out from Dirac's large-number hypotheses which relate to the ratio of electric force to gravitational force and the ratio of the age of the universe to the atomic time unit associated with atomic processes, we present in $\$ 2$ this new equation of quantum cosmology which operates the reconciliation between the macrocosm and the microcosm. In $\$ 3$ and $\$ 4$, we discuss the irreversible cosmological time resulting from the equation, as well as the role of the mass (heavy) relative to the gravitational constant G. In $\$ 5$ we discuss the links that the equation establishes between gravitation (structure of condensation) and electromagnetism (structure of expansion), between relativity and quantum theory. We apply the formula to Planck's time. We speak of the new essential variable $M_{v p}^{2}$, and briefly of a new principle, the principle of compensation. In $\$ 6$ we discuss the negative energy solutions banned by physics, and we deplore that half of the universe escapes us. We present the electro-gravitation in $₫ 7$, from the equation which repre-
\end{abstract}


sents a super hydrogen atom. In $\$ 8$ we show that the global mass (gravitational) is variable: it increases during the expansion while the mass of the elementary particles decreases. In $\$ 9$ we approach the spontaneous symmetry breaking; when it occurs, the arrows of the equation are momentarily reversed: such a mechanism would apply to the Allais effect, also mentioned in $\$ 6.4$. $\$ 10$ and $\$ 11$ deal with the energy linked by the equation to matter through expansive space-time. The equation transforms electromagnetic kinetic energy into a gravitational mass, considered as a potential energy. Entropy increases according to the arrow of time towards the future. In $\$ 12$ we discuss of the prevailing theory of inflation. We note the similarity between the proclaimed acceleration of current expansion and inflation. Physicists have interpreted the positive cosmological constant in terms of vacuum energy which would be $10^{120}$ times higher than the dark energy density deduced from the astronomical measurements. However, the high theoretical value of the vacuum energy (and the cosmological constant) has no observable pending in the cosmos. In $\$ 13$ we suggest that these several orders of magnitude difference problem are solved by the theory of relation, which indicates a deceleration of the expansion. Finally, in $\$ 14$, we close by speaking of a model of cyclic universe and about the object of this paper, a dynamic equation that allows to build a quantum cosmology.

\section{Keywords}

Theory of Relation, Quantum Cosmology Equation, Irreversible Cosmological Time, $\pi$, New Variable, Negative Energy

\section{Introduction}

\subsection{History of Unifications}

Historically [1], Newton's discovery of the law of gravitation can be appreciated as the first "unification", combining the laws of heaven and earth. The next great leap took place in the mid 1860's with Maxwell's theory of electromagnetism uniting electricity and magnetism. In 1905, Einstein created the special theory of relativity connecting space and time and associating the concepts of matter and energy. In 1915 , he proposed general relativity, which explained gravitation as the marriage of space-time and matter-energy. In the 1960's, the works of S. Weinberg, A. Salam and S. Glashow led to the unification of the electromagnetic interaction and the weak nuclear interaction. The next step, namely the unification of the electroweak and strong interactions, drove to the electronuclear theory (GUT) whose predictions were the object of no conclusive result. As for the ultimate synthesis-the unification of gravitation and GUT-it has defied all attempts.

\subsection{Dirac's Conjecture}

More than seventy years ago, Paul Dirac suggested that more than a coincidence was at work between the age of the universe in atomic time units and the ratio of 
the electric force between an electron and a proton to the gravitational force between the two $\left[k e^{2} /\left(G M_{p} M_{e_{-}}\right)=10^{40}\right]$ [2] [3]. The most fundamental unit of time would be one associated with atomic processes, because it would depend only on basic natural constants, such as the electric charge (e), the mass of the electron $\left(M_{e^{-}}\right)$, or the speed of light (c). This time unit, which appears throughout physics as the basic time scale for atomic and nuclear processes, is roughly the time required for light to travel the electron radius: $10^{-15} \mathrm{~m} / 10^{8} \mathrm{~s}=10^{-23} \mathrm{~s}$. Thus the evaluated age of the universe $\left(10^{17} \mathrm{~s}\right)$ in atomic time units is $10^{17} \mathrm{~s} / 10^{-23} \mathrm{~s}=10^{40}$. Dirac postulated that the near equality of these two numbers was a manifestation of some as yet the unknown deeper law of nature that required them to be nearly equal for all time. The problem is that the age of the universe is increasing. If the quantity between the two $10^{40}$ is to be maintained, then one of the other numbers must change with time. For many physicists, the gravitational constant $(G)$ seems the only plausible candidate which can vary in spite of general relativity, which states that $G$ is a physical constant whose numerical value is fixed.

Our reflections and insights on this issue have made us discover the theory of Relation (let us understand well: the word "relation" means connection, link, and it is not excluded that it means "unification") whose mode of action decrypts the order of the cosmos. It proposes a model of quantum cosmology through an equation that builds a relationship between electromagnetism, Newtonian gravitation, special relativity, quantum physics and general relativity.

\section{Equation of the Theory of Relation}

\subsection{The Equation}

Let us compare the electrostatic and the gravitational forces between two protons in the same nucleus, with a distance of 0.2 nanometers [4]. We will use the MKS system which has the advantage of incorporating the constants of the permittivity of free space and of permeability of free space. The value of the Coulomb constant $k$ is $1 / 4 \pi \varepsilon_{o}=8.9875 \times 10^{9} \mathrm{~N} \cdot \mathrm{m}^{2} / \mathrm{coul}^{2}$. The value of the constant $\varepsilon_{o}$, called permittivity of free space, is $8.8542 \times 10^{-12} \mathrm{coul}^{2} / \mathrm{N} \cdot \mathrm{m}^{2}$. According to Coulomb's law, the electrostatic repulsive force is $F_{e}=q^{2} /\left(4 \pi \varepsilon_{0} R^{2}\right)=5.775 \times 10^{9} \mathrm{~N}$; $e^{2} /\left[4 \pi\left(8.8541878 \times 10^{-12}\right)\left(0.2 \times 10^{-9}\right)^{2}\right]$. The attractive Newtonian force is $G M_{o p}^{2} / R^{2}=4.666 \times 10^{-45} \mathrm{~N}$. The ratio is $F_{e} / F_{g}=k e^{2} / G M_{p}^{2}=1.23 \times 10^{36}$.

Let us pursue Dirac's suggestion on the time, and replace the ratio by a universal time factor with the constants $G$ and $c . F_{e} / F_{g}=t_{o} c / G ; F_{e}=F_{g} t_{o} c / G$. And suppose we relativize the masses of the protons, in accordance with special relativity, as if they were moved with a speed of $200,000 \mathrm{~km} / \mathrm{s}$, we would obtain

$$
\begin{aligned}
& k e^{2} /\left[R_{o}\left(1-v^{2} / c^{2}\right)^{\frac{1}{2}}\right]^{2} \\
& =G\left[M_{o p} /\left(1-v^{2} / c^{2}\right)^{\frac{1}{2}}\right]^{2} /\left[R_{o}\left(1-v^{2} / c^{2}\right)^{\frac{1}{2}}\right]^{2}\left[t_{o} c / G\right],
\end{aligned}
$$


thus we would have

$$
k e^{2}=M_{V P}^{2} t_{o} c .
$$

[ $M_{o p}$ is rest-mass; $M_{o p}\left(1 /\left(1-v^{2} / c^{2}\right)^{1 / 2}\right)$ gives $M_{v p}$, i.e., rest-mass + kinetic energy (T); $v=200,000 \mathrm{~km} / \mathrm{s}=2 / 3 c$ ].

Note that in this model, the speed of the relativized protons is identified with the estimated speed of the recession of galaxies and that it determines all other variables. We found reasonable to adopt the speed $2 / 3 c$. Since this is dependent on astronomical observations which are constantly evolving, the speed will be adjusted accordingly.

Particles come in pairs, each with a counterpart antiparticle

$$
\begin{gathered}
\pm k e^{2}= \pm\left[M_{o p} /\left(1-v^{2} / \mathrm{c}^{2}\right)^{1 / 2}\right]^{2} t_{o} c \\
2.3 \times 10^{-28} \mathrm{~kg} \cdot \mathrm{m}^{3} \cdot \mathrm{s}^{-2}=\left[\left(1.672 \times 10^{-27} \mathrm{~kg}\right) /(1-4 / 9)^{\frac{1}{2}}\right]^{2}\left(1.528 \times 10^{17} \mathrm{~s}\right)\left(3 \times 10^{8}\right) \\
2.3 \times 10^{-28} \mathrm{~kg} \cdot \mathrm{m}^{3} \cdot \mathrm{s}^{-2}=\left(2.2439 \times 10^{-27} \mathrm{~kg}\right)^{2}\left(1.528 \times 10^{17} \mathrm{~s}\right)\left(3 \times 10^{8}\right) .
\end{gathered}
$$

We ascertain that the link between the charge squared and the relativized proton's mass squared confers a universal time of $1.5283 \times 10^{17} \mathrm{~s}$ multiplied by c. That time gives 4.84 billion years $\left[\left(1.5283 \times 10^{17}\right) /(365.24 \times 24 \times 60 \times 60)\right]$.

\subsection{Linear Time and Transverse Time}

This time for a speed of $200,000 \mathrm{~km} / \mathrm{s}$ gives a time well below the 13 to 15 billion light-years expected. Relativized protons should be with a much lower rate to obtain these ages. Therefore, the radius $t_{o} c$ of about 15 billion light-years could be confused with both an electromagnetic wave and a gravitational wave. The two are transverse. However, since we assimilate the speed of the relativized protons to the velocity of recession of galaxies and that the latter is not evaluated less than $2 / 3 c$, we exclude this possibility.

The other alternative is to consider that the cosmological time of Equation (2) is a mathematical "linear time" that evolves as a "longitudinal" ray of light. A point particle on a cosmological space-time ray will go straight ahead, such as a Euclidean space-time line, from one point to another. It will cross the radial radius $t_{o} c$ (=A-Z), in 4.84 billion light-years. This radius can then become confused with a longitudinal gravitational wave or a longitudinal electromagnetic wave. Although we do not know these waves, they cannot be forbidden in theory. We believed for a long time that sound waves were exclusively longitudinal while they are also transverse.

Linear time, that is to say, the time required for a particle to travel a distance $\mathrm{A}-\mathrm{Z}$ in a straight line as would make a longitudinal wave, matches with 15.21 billion years in circular time, or linear time multiplied by $\pi$. The circular time expresses the required time for a particle can travel the same length in rotating around the line, as would a transverse wave [5]. 
One can imagine that a wave rolled up around the radial line A- $Z$ would travel it in the 5.21 billion years, which is linear time multiplied by $\pi$. It fits a transverse electromagnetic wave

$$
k e^{2}=M_{V P}^{2}(\pi) t_{o} c .
$$

Of this expression, one must keep in mind that $\pi$ is used for winding the particle spirally around the radial length $t_{o} c$. It could be a transverse electromagnetic wave but it could also be a transverse gravitational wave. Mathematically, the equation should be

$$
(\pi) k e^{2}=M_{V P}^{2}(\pi) t_{o} c .
$$

From a physical point of view, we prefer however the Equation (4), because it allows highlighting, for the same distance, the transverse character (and thus electromagnetic) with respect to the linear character. It can be said that the second of the longitudinal wave is worth the second of the transverse wave divided by $\pi$. It means that a particle will browse transversely a distance $A-Z$ while in the same time another particle will cover a radial distance $A-Z \times \pi$. We estimate that it is adequate and corresponds roughly to the observations. The photon is the boson of the transverse electromagnetic wave of space-time.

It has been shown that space-time is electromagnetic (vacuum minimum energy, etc.). So, using the basic unit of time, the second, considered electromagnetic, the universe was estimated at about 15 billion light-years. Our equation, calculated for a relativistic velocity of $2 / 3 \mathrm{c}$ for the protons, gives 15 billion light-years using $\pi$. We estimate that it is adequate and consistent with the observations. The photon is the boson of the transverse electromagnetic wave of space-time.

If this transverse electromagnetic wave was unfolded into a longitudinal electromagnetic wave, the distance $\mathrm{A}-\mathrm{Z}$ would be multiplied by $\pi$, what would give 47 billion longitudinal light-years. We would have, always at the speed of light, distances at least three times the length of the distances presently estimated. The seconds of this longitudinal electromagnetic wave would have a time three times shorter than the seconds of the transverse wave.

The particles that may be associated with longitudinal electromagnetic waves and longitudinal gravitational waves could be the neutrino and the graviton. The longitudinal electromagnetic wave already exists. The gravitational wave, which has always been considered to be transverse, would have been captured in 2015 . This does not exclude the existence of a longitudinal gravitational wave [6].

\subsection{Formula of the Expansion}

We know that nucleons are forming $99.97 \%$ of the known matter. Neutron and proton are two states of the nucleon. Suppose that the protons of our equation are galaxies which move away at $2 / 3 c$, then we get a "cosmological equation" that determines the age and distance of the universe in relation to the speed of recession of galaxies. The more the speed of recession of distant galaxies de- 
creases (velocity $\sim c^{2} / c^{2}$ at the beginning toward lower speeds: $v^{2} / c^{2}$ ), the more they move away from the Earth and the more the age of the universe increases. Velocity, distance, and age are correlated for the first time in an equation. The flight of the galaxies at $2 / 3 c$ corresponds to 15 billion years. Both numbers are roughly consistent with the real estimates of science.

The Equation (4), in the form

$$
\pm(\pi) k e^{2}= \pm M_{V P}^{2}(\pi) t_{o} c
$$

is the equation of the expansion. It establishes a clear mathematical link between electromagnetism and gravitation. The term " $(\pi) t_{0} c$ ” corresponds to the radius of the universe. To the right-hand side, matter $\left(M_{V P}^{2}\right)$, space and time $\left(t_{o} c\right)$ are mathematically related into one whole.

It can be seen in the Equation (1) that the expression

$G\left[M_{o p} /\left(1-v^{2} / c^{2}\right)^{1 / 2}\right]^{2} /\left[R_{o}\left(1-v^{2} / c^{2}\right)^{1 / 2}\right]^{2}$ links Newtonian gravitational force and relativity. We get a relativized Newtonian gravitation, which means, firstly, that gravity is a reality everywhere and, secondly, that special relativity is not only a mathematical utility, not just a Galilean reference frame without gravity. Both are linear theories applied to a tri dimensional Euclidean geometry with linear-plan space-time. These are applied to a linear two-dimensional Euclidean space-time with flat linear theories. In addition, electromagnetism is also a linear theory.

In Newton's theory of gravitation, the force $G M m / r^{2}$ transmits instantaneously an energy or a signal. Newton was unhappy with the instantaneous or "action at a distance" phenomenon associated with gravity. Poincaré (1904), Minkowski (1908), and de Sitter (1911) agreed that gravity must propagate with the speed of light. For according to special relativity nothing moves faster than light, not even gravity. None of the several gravity theories-even from Einstein, that was consistent with special relativity in that the speed of the propagation of gravity was the speed of light-was satisfactory. In the proposed equation, the combination of the Lorentz transformation and $t_{0} c$ ensures that the velocity of light or gravity does not go faster than the velocity of the universal constant $c$ [7].

According to the theory of Relation, gravity is not a separate force, but an aspect of electromagnetism. Both forces are joined by the four-dimensional space-time. In fact, the gravity results from the dissolved energy of the expanding electromagnetism which does not stop creating the space-time. Deep down, electric and gravitational forces are part of a common superforce: electro-gravitation. Like magnetism and electricity are two sides of electromagnetism [8]. In subatomic particle physics, electro-gravitation takes the aspect of the electrostatic force, and the gravitation force, $10^{36}$ weaker, plays no direct role there. When the pair of particles with both positive electric charges which repel and move away at nearly the speed of light, creating the "space-time" between them, electromagnetism decreases with distance and takes the name gravity. On the large scale, electro-gravity becomes gravity. We are led to think of the gravi- 
tational forces as being electromagnetic forces with attractive charges acting in space-time instead of in the subatomic world. The driving force of expansion, caused by the initial explosion, would result from the repulsive charges of the electromagnetic forces operating in the universe [9].

\subsection{Other Relativistic Expressions}

Here are other relativistic expressions that express the mass variation of a moving proton as a function of velocity and cosmological time. They do not take account here of $\pi$ as if $t_{o} c$ was longitudinal and not transverse. The same applies to the rest of the paper.

( $M_{o p}$ is the rest mass; $M_{o p}\left(1 /\left(1-v^{2} / c^{2}\right)^{1 / 2}\right)$, or $\Delta M_{p}$ is the kinetic energy $T ; M_{v p}$ is the relativized mass with kinetic energy; $v=2 / 3 c$ )

$$
\begin{gathered}
k e^{2}=M_{o p}+\left\{M_{o p}\left[\left(1 /\left(1-v^{2} / c^{2}\right)^{1 / 2}\right)-1\right]\right\}^{2} t_{o} c \\
2.3 \times 10^{-28}=\left[\left(1.6725 \times 10^{-27}\right)+\left(5.71 \times 10^{-28}\right)\right]^{2}\left(1.52 \times 10^{17} \mathrm{~s}\right)\left(3 \times 10^{8}\right),
\end{gathered}
$$

or

$$
\begin{gathered}
k e^{2}=\left(M_{o p} \times \Delta M_{p}\right)^{2} t_{o} c \\
2.3069 \times 10^{-28}=\left[\left(1.6725 \times 10^{-27}\right)(1.3416)\right]^{2}\left(1.5283 \times 10^{17} \mathrm{~s}\right)\left(3 \times 10^{8}\right) .
\end{gathered}
$$

Relativistic expression for kinetic energy of a moving proton (quantum mass)

$$
T=M_{v p} c^{2}-M_{o p} c^{2}=M_{o p} c^{2}\left[\left(1 /\left(1-v^{2} / c^{2}\right)^{1 / 2}\right)-1\right] .
$$

Potential energy for the electrostatic field of point charges united to potential energy for the gravitational field

$$
\begin{aligned}
& -k e^{2} /\left[R_{o}\left(1-v^{2} / c^{2}\right)^{1 / 2}\right]^{2} \\
& =-\left\{G\left[M_{o p} /\left(1-v^{2} / c^{2}\right)^{1 / 2}\right]^{2} t_{o} c\right\} /\left\{\left[R_{o}\left(1-v^{2} / c^{2}\right)^{1 / 2}\right] G\right\} .
\end{aligned}
$$

Formula of the total energy of the proton in function of $e, c$, and $t_{o}$

$$
\begin{gathered}
k e^{2}=M_{V P}^{2} t_{o} c ; M_{v p}=\left(k e^{2} / t_{o} c\right)^{1 / 2} ; M_{o p}=\left(1-v^{2} / c^{2}\right)^{1 / 2}\left(k e^{2} / t_{o} c\right)^{1 / 2} \\
E=M_{o p} c^{2}+T=M_{v p} c^{2}=\left(k e^{2} c^{3} / t_{o}\right)^{1 / 2} \\
E=M_{o p} c^{2}=\left[\left(1-v^{2} / c^{2}\right)\left(k e^{2} / t_{o} c\right) c^{4}\right]^{1 / 2}=\left(1-v^{2} / c^{2}\right)\left(k e^{2} c^{3} / t_{o}\right)^{1 / 2} .
\end{gathered}
$$

This is for the fermion. New formula of energy for the boson $\left(m_{o}\right)$ is

$$
\begin{gathered}
E=m_{o} c^{2}=M_{v p}^{2} h c / k e^{2} \\
k e^{2}=M_{V P}^{2} t_{o} c ; k e^{2}=M_{V P}^{2} h / m_{o} c ; k e^{2}=M_{V P}^{2} h c / m_{o} c^{2} .
\end{gathered}
$$

This means that electricity is a manifestation of energy like energy and matter are equivalent. Proton (and neutron) and electron are grains of electricity. The masses of proton and electron have EM origin: $M_{v p}=\left(k e^{2} / t_{o} c\right)^{1 / 2}$, and the mat- 
ter is composed of grains of electricity. Matter atom is done of a multitude of elementary particles that are electricity, so properties of matter must be explained by properties of electricity.

On the basis of $k e^{2}=M_{V P}^{2} t_{o} c=M_{V P}^{2} 2 G M^{o} / c^{2}=M_{v p}^{2} 2 G M^{o} c^{2} / c^{4}$, the new formula of the energy of the ordinary matter of the universe will be

$$
E=M^{o} c^{2}=k e^{2} c^{4} /\left(M_{V P}^{2} 2 G\right) .
$$

\section{Cosmological Time}

The Equation (2) $\left[k e^{2}=M_{V P}^{2} t_{0} c\right]$ is written with a real and cosmological time (neither relative nor absolute) that governs the infinitely small and the infinitely large. It allows considering time as a real entity which contains in itself the difference between the past and the future. It can open a new chapter of the physics because so far, the more the physical theories have been developed, the more the notion of time has become evanescent. First with the introduction of relativity in 1905 which made it lose its absolute character; the notions of present or of duration turning dependent on the observer; an overt indifference where time becomes intimately linked to space for which this distinction has no meaning. Then with quantum mechanics, two decades later, who dived in the "fuzzy" the idea of time. The laws of microphysics show no preferred flow direction. At such an extent that for physicists if a broken glass is never spontaneously repaired, it is not because we cannot go back in time, but simply because the configuration "repaired" is less probable than "broken" [10].

Nevertheless, in the cosmological theories of Einstein, de Sitter and Lemaittre, new ideas related to the character of universal space had been introduced, but no corresponding progress was achieved regarding the idea of time, except to the extent that the phenomenon of expansion tended to suggest a finite past, rather than an infinite past. Just as Einstein did make the progress that is known by analyzing concepts like that of simultaneity, similarly the next progress of physical theory will be obtained by taking again the analysis of time to the point where he left it.

The cosmological time found in the equation would be the age of the universe. The reference is the beginning of space-time from the Planck time. It would recover a character not absolute as before the relativistic revolution, but universal that would integrate the difference between the past and the future with the acquirements of this revolution that unites time and space. The energy associated with the immateriality and the mass associated with ordinary matter could be stored in two opposite and complementary structures. This "new" time and this new energy and mass ratio would redefine general relativity, making it global and compatible with the quantum rules [9].

If we admit that the universe is a kind of expanding super-atom that gives the age of the universe, then we have an arrow to the future which is the same of at least three arrows of time that distinguish the past from the future: thermodynamics (disorder increases) cosmological (universe expansion rather than contraction), psychological (we remember the past, not the future) [11]. The flat 
space-time, i.e., zero curvature, makes the universe seem as being very close to the special case, intermediate between open and closed universe. The closed universes collapse eventually, and may then undergo further cycles of expansion and collapse, like a bouncing balloon. A universe closed by gravity is the equivalent of a black hole. We assume that the universe is at the highest of his jump and is about to plunge forward, which means that the cosmological time continues by orienting towards the big crunch, while the thermodynamic time passes from cold to hot.

In any event, the colossal amount of kinetic energy contained in the original proton varies with time. In fact, energy relieved by two energetic protons which move away from each other engenders the radius of the universe. The term " $t_{o} c$ " corresponds to the radius of the universe and represents the cosmological time linked to the universal thermodynamics. We say that this cosmo-thermodynamics time, that shapes the radius of the universe, stems from two charges in the form of relativized protons which run away from each other creating so the space-time of this universe. One could say that the pairs of protons go away simultaneously from the center in all directions and two opposite directions constitute geometrically the diameter. This simultaneity suggests another time behind the thermodynamic time that we could call "duration". The velocity v of the Lorentz transformation indicates both the speed of the proton which decreases with expansion and its remaining mass (rest + kinetic energy). The released energy is propagated with velocity $c$, as an electromagnetic wave, which suggests that the frequency decreases with time [12].

\section{The "Flow" of Time and the Universal Constant $G$}

Lancelot L. Whyte in a short essay, Archimedes or the Future of Physics (1927) [13], pointed out that in each of the two major physical theories of the twentieth century, the fundamental role was played by a particular natural constant: In relativity by $\mathcal{c}$, the velocity of light in vacuum, and in quantum theory by h, the Planck constant. He suggested that the next progress would be associated with a new fundamental constant that would concern the flow of time. The idea that time can be an active factor of causality means from the mathematical point of view that $t$ must appear in the expression of the law. Such a law would express the fact of the historical and irreversible duration, or the "flow". The irreversible flow of time (cosmological time), linked to the irreversible phenomenon of expansion and our consciousness of the one-way flow of time, becomes a necessary element of any theory of the structure of nature. Thus, while the first principle of thermodynamics, that of conservation of energy, concerns time only as a simple "duration," the second principle implies the idea of flow. This notion of flow is fundamental and we consider that the expansion of the universe is its supreme manifestation.

Based upon the fundamental unit of time and on the fundamental physical constants, this cosmological time confirms the intuition of Paul Dirac, namely 
that the dimensionless number $10^{40}$ is not a constant but a variable of time in relation to the age of our universe. He thought that this number was determined by particle physics and also by the gravitational influence of the entire universe. In 1937 and in 1938, he proposed that $G$ varies like the inverse age of the universe, so as the universe expanded from the big bang, the gravitational constant, or force, became weaker and weaker as time passed until today when we experience the present very weak force of gravity. But he was unable to apply it to Einstein's gravity theory [14].

For our part, we postulate that the gravitational mass of the universe varies proportionally to the age of the universe [7] [15]. If we assert that the electric forces and the gravitational force which are exerted between two particles of identical mass are disproportionate, the gravitational interaction is reduced to almost nothing. We have previously (section 2.1) replaced the numerical difference $10^{36}$ (for protons) by the factor $\left(t_{o} c / G\right)$. Although Dirac has concluded that it is $G$ which varies with time, we note that $G$ is excluded from the equation, just like for special relativity: $\left[k e^{2} / r_{v}^{2}=\left(G M_{v p}^{2} / r_{v}^{2}\right) /\left(t_{o} c / G\right)=k e^{2}=M_{V P}^{2} t_{o} c\right]$. It is the mass $M_{v p}$ which varies rather than the constant $G$. In fact, even if $G$ retains its status of an invariable constant of nature, as for general relativity, it turns out to vary through his substitute, the mass, which is amended from time. This confirms the intuition of Dirac and entails a modification of the gravity leading to a new kind of cosmology in which it is as much the mass of the particles as the mass of the whole universe which changes with time.

Ultimately, the theory of Relation considers that the mass of elementary particles changes with time (we do not speak here of the naked mass which remains invariant), that $G$ is a fixed constant of the nature and that the space-time of the theory of Relation $\left(t_{o} c\right)$ is almost the same as that of special relativity $(t c)$ : a four-dimensional Euclidean space-time continuum [11], except that he contains an electromagnetic aspect.

\section{Link between Quantum Mechanics and General Relativity}

\subsection{Theory of Relation}

According to the theory of Relation [16], the classical gravitation is almost zero at the Planck time because its energy is entirely in the potential state, the inverse of the kinetic energy of the electromagnetic interaction. Thus in equation

$$
k e^{2}=M_{V P}^{2} t_{o} c=2 G M^{o} / c^{2},
$$

the kinetic energy of the squared mass of the relativized proton $\left(M_{v p}^{2}\right)$ decreases in an inversely proportional way to the time and to the mass of the ordinary matter $\left(M^{o}\right)$. The time generated by the expansion is inversely proportional to the quantum mass which decreases and proportional to the ordinary mass which grows, while we keep $G$ of general relativity as a fixed constant of nature. Although $t_{o} c$ looks like the Euclidian four-dimensional space-time continuum of special relativity [11], it is dissociated by its irreversibility and its electromag- 
netic aspect.

If we write $k e^{2}=M_{V P}^{2} t_{0} c=M_{V P}^{2} h / m_{o} c$, the cosmological time which extends from $10^{-43} \mathrm{~s}$ to $10^{17} \mathrm{~s}$, associated with $h$ and $c$, reveals the quantum structure of space-time itself, which means a limit to the divisibility of space and above all to the divisibility of time.

One can imagine big implications if we write

$$
k e^{2}=M_{V P}^{2} t_{o} c=M_{V P}^{2} h / m_{o} c=M_{V P}^{2} 2 G M^{o} / c^{2} .
$$

\subsection{Structure of Expansion and Quantum Theory}

In this proposed model of quantum cosmology, there are two associated structures (expansion and condensation) of the world and a time scale incorporated into two different theories, quantum mechanics and relativity, each of which is of fundamental importance. In

$$
\pm k e^{2}= \pm\left[M_{\text {op }} /\left(1-v^{2} / c^{2}\right)^{1 / 2}\right]^{2} h / m_{o} c= \pm\left[M_{o p} /\left(1-2^{2} / 3^{2}\right)^{1 / 2}\right]^{2} h / m_{o} c
$$

or

$$
\pm k e^{2}= \pm M_{V P}^{2} h / p,
$$

we associate electromagnetism, Newtonian gravitation, special relativity and quantum physics. The formula $t_{o} c=h / m_{o} c$ expresses the electro-gravitational field bound to $M_{V P}^{2}$.

Protons $\left(M_{V P}^{2}\right)$ represent the stable particles of matter of the expanding universe. They move in every given direction at a speed less than $c$. So at any epoch posterior to the Planck era, the system fills the inside of a Euclidian sphere of radius $t_{o} c$. The nearest particles from the center, which emerge from the Planckian era, seem to move away with a speed $v$ very close to $c$. At the beginning, their rest mass is covered with a huge kinetic energy. The speed of the expansion decreases and the kinetic energy of protons are subject to a transformation into a sort of frozen energy, in bodies with mass. We suppose arbitrarily that the speed of the particles of the current universe is $2 / 3 c$ [17]. The proton-likened to a galaxy because it consists of protons-will be livened up at the most in a uniform motion at a constant speed when he will have exhausted almost all its kinetic energy and will be close to its rest mass $\left(1.6725 \times 10^{-27} \mathrm{~kg}\right)$. It is a "zero" speed motion and this could mean that the expansion will be replaced by a contraction into a new region that did not exist before and that the universe will go towards a new space-time bounce [18].

By uniting $E=m_{o} c^{2}$ of the relativity with $E=h v$ of the quantum theory, we obtain $t_{o} c=h / m_{o} c$. This field produces energy packets which are bosons. The particle mediator $m_{0}$ represents as much the quanta-photon as the quanta-graviton. The latter (we are talking about the graviton from the big bang) carries the gravity and is similar to the photon of electromagnetism. Both are moving at the speed of the light, have energy but no rest mass. Their paths can be represented by straight lines in Euclidean space. If we talk of an electromag- 
netic field for the photon, we speak of an electro-gravitational field for the graviton. The electro-gravitational wave " $t_{0} c$ " could belong to the family of electromagnetic waves. A de Broglie wave is associated with their motion and is affiliated with the speed of the proton.

\subsection{Structure of Condensation and General Relativity}

The term $G M^{o} / c^{2}$ represents the structure of condensation, and general relativity in a more global than local aspect. Einstein saw a possibility to obtain a geometrical interpretation of gravitational forces analogous to centrifugal forces. Centrifugal forces and gravitational forces are proportional to the mass of the body which they are applied [19]. The equivalence between inertial, gravitational and centrifugal forces is $F=m a=G M m / r^{2}=m v^{2} / r$. Radius is $G M^{o} / v^{2}$. With velocity $c, r=G M^{\circ} / c^{2}$. This expression of the radius of general relativity is, in fact, the Schwarzschild radius. In Equation (4), we said that $t_{0} c$ was the radius of the universe. So $R=t_{o} c=G M^{\circ} / c^{2}$. For reason of symmetry, we will take $2 \pi G M^{\circ} / c^{2}$ as the relevant universal radius. Assuming a relativistic cosmic speed of $2 / 3 c$, the mass of the present universe will be

$$
\begin{gathered}
\pm k e^{2}= \pm\left[M_{o p} /\left(1-v^{2} / c^{2}\right)^{1 / 2}\right]^{2} 2 \pi G M^{o} / c^{2} \\
\pm 2.3069 \times 10^{-28} \mathrm{~kg} \cdot \mathrm{m}^{3} \cdot \mathrm{s}^{-2}= \pm\left(2.2439 \times 10^{-27} \mathrm{~kg}\right)^{2} 2 \pi G M^{o} / \mathrm{c}^{2} .
\end{gathered}
$$

We then obtain $M^{o}=9.82 \times 10^{51} \mathrm{~kg}$. Because the mass is related to $t_{o} c$ and that $t_{o}$ is a "linear time", the mass is $9.82 \times 10^{51} \mathrm{~kg} \times \pi=3.08 \times 10^{52} \mathrm{~kg}$. This is approximately the estimated mass of the universe, which tends to confirm the version of Mach's principle incorporated into Einstein's theory [20].

According to this, the structure of space-time depends on the average distribution of all matter in the universe. And inertia of an object depends on the structure of space-time. Einstein's equations produce the adequate Machian influences in a closed universe in which there is enough matter to gravitationally bend space on itself.

The expression $2 \pi G M^{\circ} / c^{2}$ means that the radius of the universe must be compressed so that the escape velocity is equal to the speed of light [21].

\subsection{Planck Time}

At Planck time $\left(\hbar / c=3.5177 \times 10^{-43} \mathrm{~s}\right)$, if we apply

$$
\pm k e^{2}= \pm M_{V P}^{2} t_{o} c= \pm M_{V P}^{2} h / m_{o} c= \pm M_{V P}^{2} 2 G M^{o} / c^{2},
$$

the mass of the "baryon-proton" $M_{v p}$ will be $1.479 \times 10^{3} \mathrm{~kg}$ $\left(2.3069 \times 10^{-28}=M_{V P}^{2} 3.51 \times 10^{-43} c\right)$.

The wavelength $\lambda=t_{o} c=R=h / 2 \pi=\hbar=1.05458 \times 10^{-34} \mathrm{~m}$. We use $\hbar$ with the Planck time and the Planck length: this is consistent with $t_{o} c$, which is linear, not circular.

With the de Broglie wave that travels at the speed of light as that of the particle $m_{o}$, the boson $m_{o}$ gives $2.09 \times 10^{-8} \mathrm{~kg}\left(k e^{2}=M_{V P}^{2} h / m_{o} c\right)$. We employ 
$h / m_{o} c$ because quantum mechanics describes a particle, not a radius.

With $k e^{2}=M_{V P}^{2} 2 \pi G M^{\circ} / c^{2}$, general relativity determines the mass of the universe at Planck time, $M^{o}=2.26 \times 10^{-8} \mathrm{~kg}$. We utilize $2 \pi G M^{o} / \mathrm{c}^{2}$ (not $G M^{\circ} / c^{2}$ ), considering that the term describes a mass with a circumference, not a radius.

Instead of having $M_{\text {Planck }}=(h c / 2 \pi G)^{1 / 2}=2.1768 \times 10^{-8} \mathrm{~kg}$, which seems to be one of two similar masses, we have $M^{o} m_{o}=h c / 2 \pi G$, which are two different masses: $m_{o}=2.09 \times 10^{-8} \mathrm{~kg}$ of quantum theory and $M^{o}=2.26 \times 10^{-8} \mathrm{~kg}$ of general relativity. The Planck mass $2.1768 \times 10^{-8} \mathrm{~kg}$ is actually the average of these two distinct masses $\left(M^{o} m_{o}\right)^{1 / 2}$. Their numerical value corresponds to Planck mass and that makes think of the famously hidden variables.

\subsection{New Variable: $M_{v p}^{2}$}

The new parameter $M_{V P}^{2}$, or $\left[M_{o p} /\left(1-v^{2} / c^{2}\right)^{1 / 2}\right]^{2}$, is an essential element. Its value changes throughout the expansion. It can be suspected of being the non-zero mean value in the vacuum of the Higgs field. It would be the scalar field of the Higgs ocean at the origin of the inertia of matter which measures the force that must be applied to an object to provide it a given acceleration. One can also conjecture that it is a hidden variable in the sense that Einstein understood it: the mass of the particle associated with the wave that would commit a serious infringement of Heisenberg's principle of uncertainty [22] [23]. It would then be possible to predict with precision any future state of the universe; the associated wave would say more than the probabilities of the particle found in different places. It could also be a mathematical tool to grasp what was really under Planck's values; at microsecond $10^{-43}$, the universe is thought to have had a size close to zero and to have been infinitely hot [11] [24]; it is the birth of the universe, but it is not the zero point of the singularity.

The Lorentz transformation of this variable [12] inscribes the equation in a relativistic cosmology (although our mathematical model is central and global whereas general relativity is above all peripheral and local). The velocity $v$ of this transformation, starting from the speed of light and moving towards 0 (it would be about $2 / 3 c$ today), constitutes a variable velocity of light. Thus the limit of a signal it was thought up till now to be that first measured with the light waves was much greater at the beginning of the history of the universe. The exchanges of heat could, therefore, be made much faster, which would have led the cosmos to have the same temperature everywhere. This would explain the remarkable precision with which the spectrum of fossil radiation appears to us today as that of a quasi-perfect black body. And that's what we are observing today. The measurements made by the COBE, WMAP and Planck satellites show that the cosmic background radiation is rather homogeneous and isotropic from the point of view of its temperature on the sky, which implies that it is the same for the density of matter. And if one attempts to understand these observations in the classical models of the expansion of the universe discovered by Friedmann, 
Lemaître, Robertson, and Walker in relativistic cosmology, one cannot succeed.

\subsection{Principle of Compensation}

The important point is that by virtue of the principle of Compensation of the theory, $m_{o}$ and $M^{o}$ are related. When $m_{o}$ decreases (as well as $M_{V P}^{2}$ on whom $m_{o}$ depends), $M^{o}$, which represents the global mass of the universe, increases. $M_{v p}^{2}$ and $m_{o}$ form the kinetic energy of the universe which decreases, whereas $M^{o}$ constitutes the potential energy that grows as heavy weight.

\subsection{A time Scale Associated with the Clocks of Two Opposing Spacelike Theories}

The cosmological time $t_{o}$ does not stop growing at the speed of rays of light and it is interpreted as the "age" of the system. The term $t_{o} c$ is at the same time the radius of the expansion and the wave of the expansion. Its metric yields the clock of the universe. It indicates that contrasting energies of particles follow the same course of time (order of $10^{60}$ between $10^{-43} \mathrm{~s}$ and $10^{17} \mathrm{~s}$, between $10^{-35} \mathrm{~m}$ and $10^{25} \mathrm{~m}$ ). The different speeds $v$ of $M_{V P}^{2}$ correspond to various values of $t_{o}$.

One can find a mathematical relation binding the times that the quantum clock and the clock of general relativity assign respectively to a series of events. This relationship can be used to compare, or convert, the epochs in one scale in epochs in the other. Unlike those who think that these two clocks have no common point, that anyway we need "mass" to build a clock by pointing out that $\mathrm{m}_{\mathrm{o}}$ has no mass, we believe we can build a clock universal without necessarily needing a rest mass [17].

You can take the content of the energy of $m_{o}$, by converting into a no rest mass or a virtual mass [18]. In this respect, we got $t_{o} c=h / m_{o} c$;

$m_{o}=4.824 \times 10^{-68} \mathrm{~kg}=2.7 \times 10^{-32} \mathrm{eV}$. The moving mass $\left(m_{o} c\right)$ pilots an associated wave, also at the speed of light $\left(t_{o} c=4.58 \times 10^{25} \mathrm{~m}\right)$ with a period of $1.5283 \times 10^{17} \mathrm{~s}$. Or $\left(\pi t_{o} c=\pi 4.58 \times 10^{25} \mathrm{~m}\right)$ with a period of $\pi 1.5283 \times 10^{17} \mathrm{~s}$. The specific frequency will be $v=m_{o} c^{2} / h$. Even if their frequencies will be below zero, and that the time between two beats of the current clock in the vacuum is as long as the age of the universe, it does not prevent building a cosmic clock that will be as significant than a clock within the ordinary matter according to the relation $t_{o} c=G M^{o} / c^{2}$.

These two clocks are interconnected due to the principle of Compensation. Thus, the particles which weigh $10^{-68} \mathrm{~kg}$ are the photon or the graviton of the empty space-time grafted to the mass of the current universe $\left(\sim 10^{52} \mathrm{~kg}\right)$. At the Planck epoch, the ordinary mass of relativity and the quantum mass of the photon (or graviton) had substantially the same value, about $10^{-8} \mathrm{~kg}$.

\section{Negative Energy}

\subsection{Ocean of Negative Energy of the Theory of Relation}

In the theory of Relation, there are more than two interconnected clocks (4) that 
allow us to speak of the universe as a sort of universal metronome. Always by virtue of the principle of Compensation, there is a transformation of so-called "negative" energy into "positive" energy. The flat space-time ocean of special relativity merges here with Higgs ocean, also assimilated with Dirac ocean, themselves amalgamated with "ether" ocean (minimal energy of the vacuum). Even if each ocean retains its specficity, misunderstood, it is part of a vast ocean, the matrix of atom and vacuum. According to our equation, there would be a transformation of a space-time more and more flat into space-time more and more locally curved. (Einstein gave his power to the idea of local curvature of space by postulating that the geometry of the universe was curved by the masses it contained and that this geometry determined the movement of material objects within it).

It is presumed that at the beginning, as much matter as antimatter were created. Why has matter triumphed? Almost all of the specialists believe that a dissymmetry would have tipped in favor of matter. We do not believe that a surplus generated by the asymmetrical reaction of the particles, during a great annihilation, would have made a tiny difference favoring a bit of matter that would have structured itself to become our world [25]. We also adopt the hypothesis of an originally symmetrical universe, but we differ on the mechanism that would have favored matter. According to us, from the initial spark, there would have been a universe shared in equal parts between positive and negative matter, but part of positive matter would have taken the direction of the nascent universe, while the negative counterpart would return to the sea of negative energy. And this progressively throughout the expansion, even today, though feebly. This transformation of negative energy into positive energy is a long quiet river and not a titanic flash of light of a fraction of a second. The universal dynamic is that energy is transformed into matter, under the impulse of a decelerating expansion that began with a speed close to that of light. The universal dynamic is that energy is transformed into matter under the impulse of an expansion which decelerates with time and which began with a speed very close to that of light. Particles and antiparticles from negative energy (Dirac sea, Higgs ocean) materialize. A separation mechanism allows antiparticles of negative energy to go in the opposite direction to the ocean to form the lands (matter), thus becoming particles of positive energy, while the positive energy antiparticles plunge into the ocean of negative energy. In conclusion, the sea of negative energy ebbs and the islands of positive matter rise. One could imagine lands that emerge while the ocean level drops.

But if it does not look that way and physicists seem to want to impose a violation of the CP symmetry which would leave an excess of matter, it is precisely because they have suppressed the negative energy.

\subsection{Invalidation of Negative Energy}

We are thus at the heart of a problem which goes back to the conflict of ether in 
the nineteenth century. Einstein stopped this war in 1905, declaring that the "luminiferous aether," the supposed carrier of light, to be unobserved, hence nonexistent. Around 1930, Dirac pointed out that the energy-momentum-mass relation

$$
E^{2}=c^{2} p^{2}+m^{2} c^{4}
$$

associated with special relativity, has two roots. It calls for both positive and negative energy:

$$
\pm E=\left(c^{2} p^{2}+m^{2} c^{4}\right)^{1 / 2} .
$$

He asked himself what to do with the negative energy solutions

$$
E=-\left(c^{2} p^{2}+m^{2} c^{4}\right)^{1 / 2} .
$$

Since all negative-energy states have lower energy than any positive-energy state, Dirac wondered why there were any filled positive states, since according to Hamilton's law all entities tend to seek the lowest-energy state. He suggested that all of the negative energy states must be filled, like the filled electron shells in the Pauli exclusion scheme. Then, unless a "vacancy" occurred, positive energy particles would "float" on the surface of the negative-energy "sea" and stay positive.

Dirac's "sea" of filled negative energy states, while it satisfied the equation, did not satisfy the physics community. Heisenberg, Pauli, Jordan and others excluded those solutions that have a negative $E$ to get over the difficulty in the classical theory. They refused the requirement of a sea of negative-energy states, insisting that theory should be based on observables alone.

\subsection{Principle of Causality Preserved with the Commutation of Spacelike Particle and Antiparticle}

It has been decreed that only positive energy is real. This certainty has been mathematically padlocked with the positive energy theorem. Rules have been established not to violate the principles of Relativity (not to exceed the speed of light) as well as the principle of causality (not to allow travel in time that would authorize backward causation on a cause that has already produced its effects), and which are compatible with quantum theory (by adding "constraints" to its formalism which guarantee that the creation of a particle necessarily precedes its annihilation). Thus, causality is expressed by means of rules of commutativity of fields operators. We speak of particles and antiparticles which must have the same mass and opposite electric charges. A creation operator $\Phi^{*}(x)$ of a particle at the space-time point $x$ and the annihilation operator of this same particle $\Phi(y)$ at the space-time point y must commute to a separation of $x$ and $y$ of the spacelike and not commute for a timelike separation. These rules prevent a particle from propagating on a spacelike line (which would mean that the particle would propagate faster than light) and, for propagation on a timelike line, that the creation of the particle preceded its annihilation. These rules can be 
satisfied only if the decomposition in plane waves of the field operators has negative frequency modes. And what do we do with these modes which, in quantum physics, correspond to negative energies, i.e., the particles that go back in time? They are reinterpreted as positive energy antiparticles that follow the normal course of time [26]. The final argument is always that negative energy is impossible, with no imaginable physical meaning.

\subsection{Validation of Negative Energy}

We believe that this is how Physics has missed half of reality. Although it seems that we live in a universe of matter without constituted antimatter, there is no reason to eliminate solutions with negative energy in quantum mechanics [27]. Furthermore, negative mass is natural in the general theory of relativity and one can exclude it only by an ad hoc assumption extraneous to the Einstein's theory.

We specify that the relation electrostatic charge-gravitational relativized mass, which is associated with a cosmological and thermodynamics time, has two roots

$$
\left[k e^{2}\right]^{2}=\left[M_{V P}^{2} t_{o} C\right]^{2} .
$$

It calls for both positive and negative universe:

$$
\pm k e^{2}=\left[M_{V P}^{4}\left(t_{o} c\right)^{2}\right]^{1 / 2} .
$$

We are convinced that the recognition of the negative energy solution can find mathematical rules in the quantum theory of fields that allow the concept of antiparticle, and that of antimatter in general, to be compatible with relativity and causality [26]. Like the solution of positive energy where the principle of causality is preserved with the commutation of "spacelike" particles and antiparticles. The original theory of Dirac would be valid. His model, according to which space is not at all empty but occupied by an infinite sea of invisible particles of negative energy, constitutes a necessary physical theory. Positive energy matter lies above this bottomless sea of negative energy states. This is in consonance with the theory of Relation, which encompasses the atom and the vacuum, and in line with its "principle of Compensation"-similar to Pauli's exclusion principle-which would greatly prohibit transitions to the sea, but would favor the transition of negative energy antiparticles from the sea towards the positive energy emerged lands.

\subsection{Allais Effect and Negative Energy}

The idea of negative mass must be taken seriously because of the desperate theoretical situation into which physics has been thrust by the anomalous behavior of discovered phenomena which cannot be explained by Newtonian gravitation and general relativity. The Allais eclipse effect is one of those. In the 1950s, Maurice Allais, interested in the influence of gravitational and magnetic fields on the movement of the paraconical pendulum, detected an exceptional deviation of the pendulum movement during the solar eclipses of 30 June 1954 
and 2 October 1959. Allais, Saxl, and Jeverdan carefully observed the behavior of three types of pendulums during solar eclipses. The pendulums exhibited significant abnormal behavior at the beginning of the phenomenon, indicating that the Moon strongly interfered with the gravitational connection between Earth and Sun at that moment. This physical anomaly, dubbed Allais effect, linked to perturbations of motion of pendulums or instruments of gravitational measurement, was also observed with varying degrees of success by others during solar eclipses [28].

The pendulums detected disturbances that sometimes indicate a drop in gravity sometimes an increase. Either a kind of antigravity, as if the involved celestial bodies lost positive energy-mass (mass $M^{0}$ decreases) or a kind of over-gravity as if they were impregnated with an increased gravity (the mass $M^{0}$ increases) [5] [29] [30]. To explain the antigravity phase, one can say that the body which is eclipsing breaks symmetry and adopts a code of behavior that belongs to a negative mass. In principle, positive mass attracts negative as well as positive mass, while negative mass repels both types of mass [31]. If masses of negative energy existed, they would behave as unexpected way as the Moon during a solar eclipse.

During the eclipse, the Moon interferes strongly with the Earth-Sun gravitational connection. In an unstable equilibrium on the point of conjunction between the curvature of the Earth which makes it its satellite and the curvature of the Sun which would make it its satellite, the Moon would then act as a negative mass. It would repel the Earth and the Sun which attract it: an anti-gravity disturbance detected by the pendulum on Earth. This conclusion could be erected as a principle that we will call the "macroscopic exclusion conjecture": The bodies which improvise themselves as a satellite around the central celestial body can only provoke repulsion, comparable to the principle of exclusion concerning the atom.

\section{The "New" Gravitational Force: Electro-Gravitation}

The equation

$$
k e^{2}=M_{v p} m_{v e-} 1836.1 t_{o} c
$$

represents the super-hydrogen atom of the universe. The proton is contained in the nucleus, while the electron rotates around the nucleus at very high velocity in a circular orbit [19] [23]. $M_{v p}$ is 1836.1 times more massive than $m_{v e-}$. The number 1836.1 indicates that the fundamental level of the hydrogen atom is in precarious equilibrium above a well of negative energy states. Dirac proposed that the principle of exclusion of Pauli forbid to an electron any transitions below the fundamental state because states were occupied by an infinite sea of invisible particles of negative energy. The empty space (1836.1 $\left.t_{o} c\right)$ is not empty. Dirac asserted that if the Pauli principle forbade transitions to the sea, nothing prevented an upward transition of the electrons from the sea to a positive energy level. This implies that particles and antiparticles can be created from an infinite 
and invisible reservoir of negative energy. There may be annihilation (the energy of their mass is conserved and transformed into photon), but it may also be that the negative energy particle returns to the sea, while its antiparticle would go in the opposite direction, so becoming a positive energy particle. There would be a continuous creation effect of positive energy. The matter would thus be incessantly created throughout the expansion [32],

$$
\begin{aligned}
& \left\{\left[M_{o p} /\left(1-v^{2} / c^{2}\right)^{1 / 2}\right]\left[M_{o p} /\left(1-v^{2} / c^{2}\right)^{1 / 2}\right]^{2} 1836.1 G\right\} /\left[R_{o}\left(1-v^{2} / c^{2}\right)^{1 / 2}\right]^{2} \\
& =k e^{2} G / R_{v}^{2} \quad t_{o} c=m_{v e-} v^{2} r_{e-} G /\left(\begin{array}{ll}
R_{v}^{2} & t_{o} c
\end{array}\right) .
\end{aligned}
$$

The gravitational force exerted by the proton on the electron of a hydrogen atom has the same magnitude as the electrostatic force of the super-hydrogen atom, in which the attraction between the positive charge of the proton and the negative charge of the electron keeps the electron in orbit around the nucleus at a distance of the order of the radius of the universe.

The two equations, $k e^{2}=m_{e-} v^{2} r_{e-}$ and $k e^{2}=\hbar^{2} /\left(m_{e-} r_{e-}\right)$, are for a hydrogen atom, with an electron that moves around a proton at an average distance of $5.29177 \times 10^{-11} \mathrm{~m}$ and at a speed of $c / 137.036$. We write $k e^{2}=M_{v p} m_{v e-} 1836.1 t_{o} c=m_{e-} v^{2} r_{e-}=\hbar^{2} /\left(m_{e-} r_{e-}\right)$.

Classical gravitational charge is $m_{e_{-}}=M_{v p} m_{v e-} 1836.1 t_{o} c /\left(v^{2} r_{e_{-}}\right)$.

Relativistic gravitational charge is $m_{v e-}=k e^{2} /\left(M_{v p} 1836.1 t_{o} c\right)$.

By substituting, $m_{v e-}=m_{e-} v^{2} r_{e-} /\left(M_{v p} 1836.1 t_{o} c\right)$, and then using $m_{e-}=\hbar^{2} /\left(k e^{2} r_{e-}\right)$, we obtain $m_{v e-}=\hbar^{2} v^{2} /\left(k e^{2} M_{v p} 1836.1 t_{o} c\right)$.

Here we have a new aspect of gravitation. In electro-gravitation, the gravitational mass is also called gravitational charge and proves to be the same thing as the inertial mass, within the principle of equivalence of general relativity [19]. Consequently, the gravitational masses have signs, as for electric charges: attraction between opposite charges and repulsion between same charges. Thus, gravitational force is not exclusively an attraction. Gravitation would be the electromagnetic force diluted by space-time. Two repellent protons in a helium nucleus, separated by a fermi [33]

$$
\begin{aligned}
\text { Em force } & =k e^{2} /\left[R_{o}\left(1-v^{2} / c^{2}\right)^{1 / 2}\right]^{2} \\
& =\left\{G\left[M_{o p} /\left(1-v^{2} / c^{2}\right)^{1 / 2}\right]^{2} t_{o} c\right\} /\left\{\left[R_{o}\left(1-v^{2} / c^{2}\right)^{1 / 2}\right]^{2} G\right\} \\
& \text { Grav. force }=G\left[M_{o p} /\left(1-v^{2} / c^{2}\right)^{1 / 2}\right]^{2}=k e^{2} G / t_{o} c .
\end{aligned}
$$

If we replace in the macrocosm the proton by a star and the electron by a body which orbits in a circle around, we obtain the Newtonian formula of the universal gravitation in relation to electromagnetism. Theoretical heresy? Einstein tried in vain to unify gravitation and electromagnetism because the electromagnetic forces are proportional to the charge and not to the mass. By assuming that 
gravitation is a manifestation of electromagnetism, maybe it will be easier to bridge the gap with general relativity, which gives a geometrical interpretation of force mathematically consistent with gravitation.

\section{Variable Global Mass during the Great Expansion}

\subsection{Cyclical Universe}

The theory of Relation advocates the concept of a cyclic universe at variance with the "increase of entropy forever", while it does not dispute that the present stars are melting away into radiation [34]. After a sufficient time, the total entropy would reach a maximum and the universe would be "heat death" [35], Electro-gravitation should then pull the entire universe back towards a final "big crunch," which is the mirror image of the initial big bang in reversed time [36]. But it is actually a forward descent that happens, like a balloon that makes leaps. In this way, the macroscopic laws would not be a reversal of the invariable time but a reversal of the thermodynamic time which would go from the cold to the warm. The story of the universe would then consist of a long journey from a big repulsive "singularity" to a big attractive "singularity". A closed universe that would have undergone a series of alternating cycles of compression and expansion.

\subsection{Variable Mass of the Universe. As the Expansion Expands, The Overall Mass of the Universe Increases While the Mass of the Elementary Particles Decreases}

The ordinary mass of the universe increases with expansion. $M^{o}$ of $G M^{\circ} / c^{2}$ increases on a cosmological timescale oriented towards the future. On the other hand, the mass of the elementary particles decreases [37]. We have seen in 5.1 that the classical gravitation is almost null at the time of Planck because its energy is entirely at potential state, in opposition to the kinetic energy of the electromagnetic interaction. And in 5.5, that when $M_{V P}^{2}$ and $m_{o}$, which form the kinetic energy of the universe, decrease, then $M^{o}$, which constitutes the potential energy, grows as a gravitational mass.

With $k e^{2}=M_{V P}^{2} t_{o} c=M_{V P}^{2} 2 \pi G M^{\circ} / c^{2}$, general relativity determines the mass of the universe at Planck time; $M^{0}=2.26 \times 10^{-8} \mathrm{~kg}$. With $k e^{2}=M_{V P}^{2} t_{o} c=M_{V P}^{2} h / m_{o} c$, the de Broglie wave which travels at speed of light, like its particle $m_{o}$, determines the boson "intrinsic" mass (or the non-rest mass corresponding to kinetic energy or energy of motion): $m_{o}=2.09 \times 10^{-8} \mathrm{~kg}$. The mass of Planck $2.1768 \times 10^{-8} \mathrm{~kg}$ is, in fact, the average of these two distinct masses $\left(M^{o} m_{o}\right)^{1 / 2}$; the former grows with the expansion to become the mass of the current universe, while the latter diminishes to become the boson of the present space-time. It is quite clear that during the expansion the mass of the elementary particles decreases in an order of magnitude $\sim 10^{60}$ and that the global mass of the universe increases by the same order of magnitude.

At Planck's time $\left(\hbar / c=3.5177 \times 10^{-43} s\right)$, the mass of the "baryon-proton" 
$M_{v p}$ is $\sim 1.479 \times 10^{3} \mathrm{~kg} . M_{V P}^{2}$ shapes a boson worth $\sim 2.17 \times 10^{6} \mathrm{~kg}$. The inverse of this number gives $\sim 4.608 \times 10^{-7} \mathrm{~kg}$, which is near the mass $\left(M^{o}=2.26 \times 10^{-8} \mathrm{~kg}\right)$ of the universe at Planck time. The assessed mass of this boson for the current universe is worth $\left(2.2439 \times 10^{-27} \mathrm{~kg}\right)^{2}=5.035 \times 10^{-54} \mathrm{~kg}$. The inverse of this number gives $\sim 1.986 \times 10^{53} \mathrm{~kg}$, which is close to the overall mass of the current universe $\left(\sim 3.08 \times 10^{52} \mathrm{~kg}\right)$. Some will see a coincidence in these numbers, where we see a connection, i.e., a coincidence that is not a coincidence.

Let us stress that the mass of the proton (or electron) is a universal constant which remains invariant whatever the epoch. What changes with expansion is not the naked mass, it is the electromagnetic energy that forms a solid mantle; this mantle gets rid of his threads throughout the time to wrap and increase the gravitational mass. In Equations (7) and (8), $\left\{M_{o p}\left[\left(1 /\left(1-v^{2} / c^{2}\right)^{1 / 2}\right)-1\right]\right\}$ and $\Delta M_{p}$ represent the kinetic energy which envelopes the rest mass of the elementary particle.

\subsection{As the Expansion Progresses, the Atomic Dimensions Raise at the Rate of the Mass of the Elementary Particles Which Diminishes}

On a cosmological time scale from the beginning towards the current age, the masses of all the elementary particles would have decreased while the atomic dimensions would have enhanced. The mass of an atom decreases with time, but its electric charge remains the same. As a result, electrons should orbit farther and farther from the atomic nucleus. The electrons would reach lower energy levels, which would require a lower energy input to dislodge them; conversely, a smaller amount of energy would be released when an electron falls into an internal orbit. The radiation emitted by a current atom would be less energetic and would have a wavelength longer than that of an atom of the past.

A body traveling a spatial length in the empty space would undergo this effect coming from that electromagnetic space itself whose wavelength increases with distance. And paradoxically, according to the principle of Compensation of the theory of Relation, this same body would undergo the effect of the global increase of the universe. This is what would explain the Pioneer anomaly. The difference between the observed trajectory and the expected trajectory of a number of not piloted space probes traveling outside the solar system or on its margins, especially the Pioneer 10 and 11 probes, would be caused by the space-time which undergoes inertia (decrease of vacuum energy) for the benefit of an increase in classic gravitation. This has allowed measuring a tiny but constant deceleration of the order of $(8.74 \pm 1.33) \times 10^{-10} \mathrm{~m} \cdot \mathrm{s}^{-2}$, as a blue-shift for probes [9].

\subsection{Cosmic Past}

Within a great contraction, the galaxies approach each other by accelerating. Inside the galaxies takes place the phenomenon of the shrinkage of the atomic di- 
mensions while the masses of the elementary particles increase. The electrons should orbit more and more near the atomic nucleus and attain higher energy levels, which would require a higher energy input to expel them. More energy would be released when an electron falls into an internal orbit; the radiation emitted by such an atom would be more energetic and would have a wavelength shorter than that of a current atom.

With the equation

$$
k e^{2} \leftarrow M_{V P}^{2} t_{0} c \text { or } k e^{2} \leftarrow M_{V P}^{2} \lambda,
$$

we can have a mathematical look back into the cosmic past. If the universe was contracting, the velocities of the protons-galaxies $\left(M_{V P}^{2}\right)$ would be reversed, so that the wavelength of the space-time wave $\left(t_{o} c\right)$ would decrease and mass of the protons-galaxies would increase. Particle and wave are equivalent and interchangeable, as are mass and energy [20] [38]. The gravitational energy is then transformed into an electromagnetic energy.

This suggests that in distant galaxies, presumed old, the atoms that emitted light would have been smaller than the atoms of the present galaxies. The wavelength of this light would be shorter and this light would be less red than that produced by the same atoms in a terrestrial laboratory. The cosmological redshift could be explained in terms of shrinkage of atoms and of the ensuing weaker reddening of light [37].

In 1998, two independent teams of astrophysicists, relying on the observation of distant type 1a supernovae, announced that the expansion of the universe did not slow down as previously thought, but was accelerating. We have already expressed our disagreement with this interpretation [9] [39]. Firstly because the determination of spatial distances in the universe is extremely imprecise. In fact, it is inconsistent to establish a link between the analysis of supernovae observations that belong to galaxies animated by movements governed by unknown equations (galaxies sometimes attracted to a galactic center, others towards the outside) and an acceleration of the expansion of space that cosmologists are unable to describe. Secondly because it could quite possibly be a case involving the assumption of temporal distances. Seeing far into space still means seeing early, according to the theory of Relation, these supernovae turned out to be less luminous and more distant than what could be deduced from their redshift because they accelerate towards the origin.

\section{Spontaneous Breaking of Symmetry and Variation of Masses}

In the equation

$$
k e^{2} \rightarrow M_{V P}^{2} t_{o} c \rightarrow M_{V P}^{2} h / m_{o} c \rightarrow M_{V P}^{2} 2 \pi G M^{o} / c^{2},
$$

the arrow indicates the direction towards the future of the great cosmic expansion. The global mass $M^{0}$ grows while the mass $M_{V P}^{2}$ and $m_{o}$ of the particles lessen.

This relation between the standard model of cosmology (that of the big bang) 
and that of particle physics gives close exchanges between these two models, what establishes a maximum fundamental state of matter counterbalanced by a fundamental state of minimum energy for space-time. The boson $M_{V P}^{2}$ can be amalgamated to a scalar boson of zero mass of a scalar field forming part of a global symmetry of the space-time continuum. The bosons would be like marbles in the channel (the bottom) of a Mexican hat. It is the true vacuum, that of the fundamental state of minimum global energy in which the field of matter does not nullify: the marbles can roll into the trench without energy spending [40].

But this symmetry at the level of the equation can sometimes be broken at the level of the solutions. Under the effect of uncontrollable fluctuations (thermal, quantum, etc.), the dynamics of a system with some symmetry temporarily reaches a state that does not have this symmetry. One can invoke this spontaneous symmetry breaking mechanism as well at the microscopic level-such as the Higgs mechanism in electro-weak unification-than at the macroscopic level, like the Allais anomaly.

When the broken symmetry is a local symmetry, the arrows in the equation are momentarily inverted

$$
k e^{2} \leftarrow M_{V P}^{2} t_{o} c \leftarrow M_{V P}^{2} h / m_{o} c \leftarrow M_{V P}^{2} 2 \pi G M^{o} / c^{2},
$$

which implies a drop in energy in the form of matter (gravitational) in favor of energy in the form of radiation. The particles of $M^{o}$ become null while the boson $M_{V P}^{2}$ and $m_{o}$ behave like marbles in the channel which are going to settle down at the top of the hat in unstable equilibrium. They are in a state of false vacuum with a higher local energy, which causes the emergence of the masses of the intermediate bosons of the electroweak unification. $M^{0}$ takes back its mass when the marbles run in the continuum of stable minimum state of energy.

A similar mechanism would apply to the Allais effect, discussed in section 6.4. The eclipse acts as an intermittent cosmological constraint that triggers a phase transition mechanism. The pendulum detects disturbances that sometimes indicate a drop in gravity sometimes a rise. Either a kind of antigravity, as if the marbles were hoisted at the top of the hat (mass $M^{0}$ decreases) in unstable equilibrium; Or a kind of over-gravity, as if they were descending to a lower level by piercing the channel, with lower energy and more gravity (mass $M^{\circ}$ increases) [5] [29] [30].

\section{Energy, Matter and Expansion}

The cosmological time of this space-time is coming from the kinetic energy of protons. It is an indicator of the energy propagated at the speed of light. The element " $t_{o} c$ " refers to a radius from the center point of a sphere created by the initial great boom (in this case, the Planck length of the Planck sphere, but it may shrink going towards the absolute zero.) Probably due to an earlier big crush, the universe began in a "cosmic fireball" and the proton was nothing else 
than a gigantic kinetic energy. This garnered energy is electromagnetic and by parting from the barrier $c$, the inertia of the particle began to fall vertiginously. When the speed went down to $99.999 \%$ of the speed of light, its mass was not more than the rest mass multiplied by 500 . The decreased speed of a thousandth entailed a division by two of the kinetic energy, and the expansion of the latter served more and more to decrease the speed. The proton, as a grain of quantum matter, is quickly emptied of most of its kinetic energy, and its rate of expansion for the present universe would be rendered to $2 / 3 c$ or $200,000 \mathrm{~km} / \mathrm{s}$. Its relativized mass is $2.2439 \times 10^{-27} \mathrm{~kg}$. The deceleration decreased inertia and moves closer to its rest mass.

The equation theoretically binds energy to matter via the expansionist space-time at the speed of light. It transforms electromagnetic kinetic energy into gravitational mass, by considering the latter as a potential energy. Such a transformation, inconceivable in the modern physics, tidies up in two well-separated categories, the mass connected to the material world and the energy associated with the immateriality. According to the equation, the same quantity of energy which decreases since the era of Planck, on one side, and the same quantity of matter which increases by the other one. Such a dichotomy is made in the context of two structures: the structure of the expansion for the kinetic energy which decreases with cosmological time, and the structure of condensation which increases over the same cosmological time to become the present universe [16].

Thus in Equation (4) $\left[ \pm k e^{2}= \pm M_{V P}^{2}(\pi) t_{o} c\right], M_{V P}^{2}$ decreases and $t_{o} c$ increases. In the equation written in the form (3)

$\left[ \pm k e^{2}= \pm\left[M_{o p} /\left(1-v^{2} / c^{2}\right)^{1 / 2}\right]^{2}(\pi) t_{o} c\right]$, the increase of quantum mass obtained by the relativization, or the Lorentz transformation, going from $v^{2}$ to $c^{2}$, is nothing else than the huge kinetic energy of the universe when it began in a "cosmic fireball."

The energy expanded creating space-time, and cooled. We can see this freezing in the formula in two ways because there are two speeds. First, the decrease of the speed of " $M_{V P}^{2}$ " cause a gradual drop in the temperature and a slowing down of the rate of expansion falls, and a concomitant increase in the gravity. These fermions, a priori in a state of radiation, were at their maximum speed until around 300,000 years. The decoupling of matter from radiation took place when the velocity passed under $c$. Second, the velocity $c$ of the electromagnetic wavelength of space-time: When the universe became the one of today, $T$ and density of radiation were reduced; " $t_{0} c$ " transports some less energetic bosons, each second contains less energy-event, and the universe as a whole does not significantly change at every second.

Our model is able to give regions of the observable universe the time to exchange heat since the big bang. Thermal equilibrium would have had time to settle and temperatures to become uniform. This allows us to refute the inflation 
phase at the beginning (with a factor of expansion and contraction of space of at least $e^{55}$ ), the proposed solution to solve the problem of causally separated regions.

According to the equation, the kinetic energy can be converted into time. Conversely, time can become kinetic energy. With relativity, mass and energy have been found to be interchangeable; now mass, energy and time can be considered as different manifestations of the same physical quantity. In this regard, time is a container of energy and has a mass. The electro-gravitational wave carries energy.

This radial movement is an electromagnetic wave. We can say that the radius of space-time belongs to the family of electromagnetic waves; the wavelength is the radius $\left(\sim 10^{26} \mathrm{~m}\right)$ of the universe and the period $\left(\sim 10^{17} \mathrm{~s}\right)$ is its age. Like in Maxwell's electromagnetic theory of light, the wave of space-time is a wave of oscillating electric and magnetic fields propagating in space [41]. We can call it an electro-gravitational wave or electromagnetic wave of space-time. It carries energy and momentum. In fact, it is the stationary electromagnetic wave, or the "background" radiation at $2.7 \mathrm{~K}^{ø}$, or the energy of the empty space. The ratio between space-time wavelength and cosmic gamma rays wavelength is $\left(10^{26} \mathrm{~m} / 10^{-14} \mathrm{~m}\right) \approx 10^{40}$.

\section{Electromagnetic Energy, Space-Time and Entropy}

The idea embodied in the equation " $k e^{2}=M_{V P}^{2} t_{0} c$ " is that the energy-mass is transformed in space-time. Space-time becomes a part of physics based on the conservation of energy, rather than an arena in which that physics takes place. The way in which the transformations work in the equation reveals that electricity, energy-matter, space-time, are inextricably linked, and should be regarded as forming our universe in a four-dimensional continuum. It implies that the principle of conservation of electricity is as important that the principle of conservation of energy [42]. The charges keep always their contents during the transformation energy-matter-space-time.

The first law of thermodynamics, the conservation of energy, is quantitative and considers the time as a simple "duration", while the second principle is qualitative and involves the flow idea. The passage of time is irreversible as much as the expansion is irreversible and suggests a finished past [17]. The second law of thermodynamics says that entropy never decreases for an isolated system. The universe as a whole has the character of an isolated system, and the law of entropy which grows in the time provides the arrow toward the future.

The growth of entropy characterizes the degradation of energy. The processes transformers of one form of energy into another are also irreversible (we are always going from "hot" to "cold"). Entropy can be written in the thermodynamic form $S=Q / T^{o}$ ( $Q$ : quantity of heat given to a system; $T^{o}$ : absolute temperature) [38] [43] [44]. If a quantity of heat does not change, and $T^{o}$ decreases, $S$ increases, meaning that the preservation of the quality of the energy quantities is 
not preserved.

We can make the equivalence with our equation:

$$
\begin{gathered}
k e^{2} / d M_{p}^{2} \rightarrow d t_{o} c \\
Q / d T^{o} \rightarrow d S
\end{gathered}
$$

The term $k e^{2}$ contains the quantity of heat, the total energy of the isolated system; $M_{V P}^{2}$ is rest mass $+T$, and $t_{o} c$ contains entropy of the empty space-time. The latter is not a vacuum but actually a plenum of particles and antiparticles being created and annihilated [32].

With time, the total entropy of the isolated system goes up moving toward a state of maximum probability. The variation of entropy is positive because the system receives heat. If we take the relation $Q=M c^{\circ} T^{o} \quad$ ( $Q$ : quantity of heat. $M$ : mass. $c^{o}$ : specific heat. $T^{o}$ : temperature), $S=Q / T^{o}=M c^{o} T^{o} / T^{o}=M c^{o}=t_{o} c$. We may say that space-time $t_{o} c$ possess a mass with a specific heat $M c^{\circ}$.

However, the energy cannot come down indefinitely. It will reach the last level of the availability and will have no more transformation capacity [34] [43]. The universe would then undergo a re-contraction in accordance with the closed model of Friedmann-Lemaitre in which space is finite and of positive curvature, and in which the expansion is decelerating.

\section{Inflationary Aberration}

\subsection{Accelerated Expansion}

The observation says that the expansion of the universe is almost offset by the gravity exerted by all matter. The universe dilates at a singular speed close to the critical line that separates the big freeze universe of the big crunch universe. We are very close to a point of balance between expansion and gravity. If all the energy released by the big bang was lower of a tiny fraction, the matter would return back and would collapse into a giant black hole. If it were slightly stronger, the matter would disperse so quickly that galaxies could not even form.

Although the world seems near its critical point, making it impossible to determine with certainty what will be its evolution, the provisional consensus is that the universe will end in big freeze, because it was found that the expansion was going by accelerating. It is postulated that there is some unknown energy that annoys gravity and causes the accelerated expansion of the universe. Einstein called the "cosmological constant" that element which could counteract the contraction caused by gravity. Rejected, then restored it is now called "dark energy". One might think officially, since 1998, that dark energy exerts a negative pressure which has the effect of causing the acceleration of the universe. But it turns out that the interpretation as much as the facts are inconclusive.

\subsection{1a Supernovae}

We have questioned (section 8.4) the interpretation of observational data in mid-1990 of type 1a supernovae ( $\mathrm{SNe} 1 \mathrm{a})$. By measuring with unequalled preci- 
sion the remains of fourteen supernovae at distances varying between 7 and 10 billion light-years, astronomers discovered that the light coming from them was $50 \%$ dimmer than it should have been according to the estimates of their distances. They interpreted the dimness as evidence that the supernovae were 10 to $15 \%$ farther away than they should have been if the expansion of the universe was slowing down. [7] The calculations obtained by the Hubble space telescope, then by telescopes located in Hawaii, Australia and Chile, were published in 1998 [45]. They too easily ruled out the criticisms which underline the decrease of the radiant energy by absorption (interstellar dust absorbs light that becomes bland), or something of the evolutionary process of the supernovae that has been misunderstood [39]. It has been suggested that the "inflection point" where the rate of expansion has ceased to decline and begin to rise under the effect of dark energy would have occurred some 5 to 8 billion years ago. Others believe that the expansion has started to speed up over 1.5 billion years ago. The acceleration of the universe has been confirmed as were the Ptolemy epicycles in the past.

\subsection{Cosmological Blunder}

We believe that the basic rules of cosmology were truncated to the point of creating a cosmological anachronism. Previous to the estimation of recessions exceeding $c$, the radial flight of the galaxies was interpreted as a process translating a general expansion of space. It has often been proposed as an example of the expansion of the space that of a rubber balloon on the surface of which are stuck confetti which represent the galaxies.

If the balloon is inflated, its surface is stretched and the confetti is moving away from each other with an apparent speed which is increased in so far as the radius of the balloon is growing. In space-time, "length" may refer to temporal length as well as spatial length. So we can receive the radiations emitted in the distant past by a system that reached speeds close to $c$, to which the expansion of space-time gives an apparent flight velocity greater than $\mathrm{c}$. This way of thinking seems long gone because it involves the center the universe [7] [43]. However, our equation claims this center of the universe. In the expression $M_{V P}^{2}$, or $\left[M_{o p} /\left(1-v^{2} / c^{2}\right)^{1 / 2}\right]^{2}$, of our equation, the speed $v$ is almost $c$, if not $c$, at the beginning, then decreases as the space-time is created. There is not an alternative. The expansion continues, the clusters of galaxies into which "matter" is distributed, are becoming more widely separated, but the rate of expansion also continues to decline from $\mathrm{c}$ towards 0 .

In astronomy, telescopes are machines to go back in time. To see far into space, we must see far in time. Further we see, weaker we see. The more a galaxy is distant, the more it moves quickly toward the big bang, primordial explosion which gave rise to the expanding universe [7]. Conversely, it decelerates as it gets closer to us [46]. But since 1998, the film of the history of the universe upside down until his first picture seems an anachronism. See far, which means see "sooner", became "later" with the telescopes. The cosmologists concluded that 
the more supernovae appear pale, the more galaxies recede from each other and accelerate towards tomorrow, towards the big freeze.

With this different astronomical vision, fundamentally spatial, whose velocity $v$ starts from 0 to increase towards $c$, the flight of galaxies is today construed as a process of acceleration of isolated systems relative to other isolated systems. For theorists, it is as if, undeniably, the galaxies were moving away from each other with speeds which are all the greater in that the distance between them is itself larger. There are galaxies which recede from us at a speed close to ninety-five percent of that of light. Based on the Doppler effect of emission-line quasars, some redshifts are so important that in applying the Hubble formula, they display some radial velocities such as our galaxy and the quasars seem to be moving apart from each other at speeds exceeding the speed of light. No matter if, in the first place, a source of radiation that would exceed the speed of propagation of light, would necessarily be invisible since, in second place, the farce of the epicycles of inflation, that continues to accelerate the universe, will necessarily render it hyperbolic [43].

\subsection{Relativity with Inflationary Sauce}

With relativity, the center is everywhere in the universe, which means that there is no real center. Strong with this a priori which excludes the irreversible time (and sometimes time itself), the reasoning of the modern astronomers led to this: the observer that I am and that I perceive as being at rest on Earth is part of a galaxy is, from that point of view, also in movement with a speed of expansion where there comes a time when it will be higher than the speed of light [18]. No matter the objection in principle of special relativity.

Since there is no center of the universe, one would think that the explanation according to which the radial escape of galaxies is the consequence of the initial explosion could not be retained. To explain the big bang, on the contrary, one will become Copernican again, and one will make general relativity to assert that space expands faster than light, because space represents neither matter nor energy. Which is senseless, because to explain the flatness of the present universe, Alan Guth suggested that the universe had ended up in a "false vacuum" at the time of the era of G.U.T. $\left(10^{-35} \mathrm{~s}\right)$, and this excited state would look like an empty space, but filled with energy. In general relativity, the energy and pressure are sources of gravitation, and a negative pressure causes a gravitational repulsion. This repulsive effect would have provoked a period of exponentially accelerated expansion. The universe would have swollen to the size of the present universe. Around $10^{-32} \mathrm{~s}$, the false vacuum would de-excite towards the true vacuum, the flatness, and all the energy of the false vacuum would have been released in the form of particles and heat. The universe would have restarted with a dark energy that would have made the flat space [32].

Apart depreciate relativity by making it appear that the false vacuum energy did not contain energy whereas the high expansion rate of the latter exceeded 
the speed of light, the theory of inflation brings an insoluble problem for the multiple theories of inflation: a dark energy that would contain the infinite kinetic energy, essential to the evolution of the hyperbolic universe, and that would have a density of $10^{122}$ times greater than that found in the empty space [45].

\subsection{Cosmological Constant: $10^{122}$ or $10^{0}$}

The experimental indications of a positive value for the cosmological constant also come from independent measurements of primordial density fluctuations observed in the Cosmic Microwave Background (CMB). The raw data from the experiences of scientific teams WMAP, COBE, Boomerang, and Maxima which have performed measurements on the fluctuations of $\mathrm{CMB}$ radiation favor the model of inflation. Inflation is a model produced from the high-energy physics, according to which the universe would have recorded, in the first fractions of a second of its evolution, a wildly high expansion rate, so that space would have become flat [45]. The teams concluded that the total density parameter $\left(\Omega_{t o t}\right)$ was $\Omega=1$ for a Euclidean universe. Both methods (supernovae and CMB) suggest that $\Omega_{m}$ (m: matter) is close to 0.3 and that it is needed to get to a flat universe, to add a contribution $\Omega_{\lambda}=0.7$, coming from a cosmological constant with a positive value. The cosmological constant plays the role of an energy density. Its value can be expressed in the same units as the density of matter, for example in grams per cubic centimeter. The $\Omega_{\lambda}$ factor is convenient for assess the contribution of the cosmological constant to the total density of the universe.

However, in the present situation, even though everyone seems to agree on the existence of a cosmological constant, its value deducted from astronomy is absolutely not compatible with that calculated by theoretical physicists. Several models are possible to calculate the value of $\lambda$ within the framework of the theories of unification, but the predicted value in most cases is $10^{122}$ times superior to the limits prescribed by astronomical observation. Dark energy should be very dense, which is the opposite of the density of the vacuum.

\subsection{Astrophysical Calculations Just for Laughs}

The dominant cosmology, starting from the cosmic microwave background radiation, considers that the big bang occurred about 13.8 billion years ago. That is an obvious dilemma since astronomers observe stars older than the universe [46]. In 1995, they had already observed them with the Hubble Space Telescope. Estimates from observations dating back to 2000 placed the star HD 140283, which lies a mere 190 light-years from Earth in the constellation of Libra, as old as 16 billion years. In 2013, it seems this star, nicknamed Methuselah, was 14.46 billion years old. Astronomers (Howard Bond, of Pennsylvania State University and the Space Telescope Science Institute in Baltimore) were puzzled because it seems the star was still older than the universe itself. Of course, they add a margin of 800 million years, meaning that the star might actually be 13.7 billion years old, barely 
younger than the universe as it is currently understood. If that pattern continues, we can expect to observe some clusters of "galaxies older than the universe" to complete the picture of the problem of "stars older than the universe" [47].

\subsection{Inflation Lobby}

There is no question to minimize the importance of data collected by the experiments since never observational cosmology has reached such precision. But the bundle of more or less plausible assumptions to extract the cosmological parameters from observational data, error bars to calculate a range of angular spectra of CMB fluctuations which are then compared to the observed spectrum, the adjustment "at best" of various parameters that can be deduced, have left the door open to biased analyses and interpretations that should have been taken with much more caution.

For some silent scientist, the fine analysis of the angular fluctuation spectrum rather suggests that the maximum likelihood is $1.2 \Omega$. The experimental balance would tip in favor of a spherical space, finite volume, although in a large radius of curvature, and ever expanding thanks to the cosmological constant. However, the lobby of inflation, strengthened by the fact that $\Omega=1$ is consistent with the data of four scientific teams, focused its efforts towards the scenario of a spectrum of fluctuations caused by inflation. The goal is to find the worst adjustments between various cosmological parameters which would give $\Omega<1$. The model of inflation has played in cosmology for over half a century the role of an original concept which became an orthodox thought. Disguised under the noble term of paradigm, it would have exercised a real intellectual terrorism to any opposite thought [45].

\section{Energy, Velocity and Cosmological Constant}

According to the theory of Relation [9] that we stand for, based on the dynamic equation of quantum cosmology, and consistent with the big bang and the scenario of the decelerated expansion, the kinetic energy of the beginning (amalgamated with negative energy and dark energy) creates not only space-time but also ordinary matter, and therefore the macroscopic gravity. Given the brute force of the big bang, the energy released at the time of creation of the universe was immeasurable. Given the brute force of the big bang, the energy released at the time of creation of the universe was immeasurable. From the first minutes, the expansion quickly converted most of this energy into ordinary matter. The energy of the primordial proton was at first moved at speed of light which has the value $\mathrm{c}$. With speeds approaching that of light, we get a Planck time $\left(10^{-43} \mathrm{~s}\right)$ and a relativized proton has a mass of $10^{3} \mathrm{~kg}$, which is a fundamentally new value in physics. Then the speed decreased gradually as the energy spread and turned into matter as we know it.

Since galaxies are moving away at a constant speed estimated $200,000 \mathrm{~km} / \mathrm{s}$, the cosmological time is about 15 billion light-years and the relativized proton 
weighs $2.2439 \times 10^{-27} \mathrm{~kg}$, according to results given by the formula above. It appears that $2 / 3 c$ is the speed where there is no longer a deceleration (from $c$ to $2 / 3 c$ ) observable. It would be close to the critical point where there seems to be an extraordinary coincidence between gravity and expansion. A speed of 100,000 $\mathrm{km} / \mathrm{s}$ would give about 26 billion light-years. The proton would then have exhausted its kinetic energy, reached its rest mass and the universe would seem moved by a movement at zero speed.

Certainly, a general dilation of the space-time would be unverifiable because we undergo it ourselves. Unless the solution is included in the cosmological constant (density measurement), the kinetic energy of the proton, which poured his energy into creating the space-time of our universe, is assimilated to a negative cosmological constant and a dark energy. It comes into play in larger scales and affects the expansion of the universe. It decreased by nearly $100 \%$, from $10^{122}$ to around $10^{\circ}$ at the present time. Because of the decreasing rate of speed, the universe expands more slowly. The kinetic energy acts as a positive pressure gas that binds the galaxies and slows the expansion. It exercises a tension which retains and brings things together, at the same time it spreads in the manner of a stretched elastic. It causes the deceleration of the universe [16] [18].

Our equation is the first to include the irreversible time. During this time, energy constitutes a variable field, very high in the phases of the primordial universe, in agreement with the calculations of physicists, but which falls very low during cosmic evolution, in accordance with the value now measured by astronomers. Dark energy (or cosmological constant) would be a "tired" energy. Bear in mind to not confuse the tired dark light with the tired white light, which stipulates that the light could have been energetically degraded and thus redshifted, during his trip through space intergalactic. However, although the light undergoes the redshifts under the influence of the Doppler effect due to the recession velocity of distant galaxies, there is no evidence that today allows to permanently eliminate the theory of tired light [37]. The theory of Relation, which combines the degraded dark light of the structure of the expansion with the shifted white light of the condensation structure, is part of the big bang theory.

We get a model of the universe that has the "temporal" behavior of closed models (in expansion-contraction), and which has the "spatial" behavior of spatially finite models. It can be likened to the oscillatory universe of Einstein-Tolman (1931), the Euclidean model of Einstein-de Sitter (1932), or the closed Friedmann-model (1922) [45] [48].

\section{Conclusions}

The equation $\left[k e^{2}=M_{V P}^{2}(\pi) t_{o} c\right]$ of the theory of Relation gives the impression of being the mathematical confirmation of the standard big bang theory, in which all the energy-matter began in an instant of time in a colossal explosion. The universe first spread to its maximum rate, then it was gradually slowed down by gravitational attraction, which now gives a sphere with a radius that is 
nearly 15 billion light-years. Deceleration can be represented by an electromagnetic space-time wave, assimilated to a negative cosmological constant, which acts at the cosmos scale as an engine of expansion (the lost energy is transformed into a gravitational matter that tends to regroup). Electricity, energy-matter and space-time are linked in a four-dimensional continuum.

We envisage cosmological history from the perspective of a cyclical universe. We saw in a previous article [9] that everything happens as if there are two universes in one. It is like if, in spite of the matter and radiation created at the big bang, there is enough excess of kinetic energy left for the pre-universe, which is slowly and automatically converted into our universe gravitation energy. Because of the inversion of the arrow of cosmological time, based on the laws of thermodynamics, the energy of the world that has contracted is negative compared to the positive energy of our expanding world. The expansion would have been preceded by a period of contraction and it would not have occurred at the same time for all the matter. In the shrinking world much of the matter contracted faster than the other, giving the big crush which is for us the big bang. Isolated pieces of matter from the contracted pre-universe would have been delayed in their appearance and development. There would have been mini bangs following the main big bang [12], a kind of violent explosive effect of strong negative pressure within a localized region where an important creative mechanism took place.

The formula introduces cosmological time into the heart of a physics that has never made the essential distinction between past and future. A cosmological, thermodynamic "space-time", from the past to the future, joins electromagnetism, Newtonian gravitation, special relativity, general relativity and quantum physics. It is important to consider this space-time as precisely the dynamic variable that allows us to consider the entire universe as the physical system where quantum nonseparability can describe as being in union two (of) uncoupled objects that have already interacted with each other, gravitationally or by exchanging radiation. In this respect, the equation finally permits a quantum cosmology.

There is still much to be said about this equation, which distinguishes three types of mass: fermionic, bosonic and that of the ordinary matter of the universe. We can find the state of the fermions in relation to the exact changing mass of the bosons, for any state of the mass-energy in the "irreversible" time. The equation is therefore in line with Yukawa's theory [20] [49] which postulates that there is an infinity of particles corresponding to an infinity of possible states of matter, and with the idea of a supersymmetry that interchanges fermions with bosons, and vice versa. In this way, this equation at once quantum and relativistic is God's equation [35].

\section{Conflicts of Interest}

The author declares no conflicts of interest regarding the publication of this paper. 


\section{References}

[1] Kaku, M. and Trainer, J. (1987) Beyond Einstein, Bantam New Age, New York, 10, 20, 21, 30, 31, 35 .

[2] Will, C.M. (1986) Was Einstein Right? Basic Books, Inc., New York, 153, 166-167.

[3] Eddington, A. (1995) Space, Time \& Gravitation. Cambridge University Press, Cambridge, 1920, 178, 179.

[4] Bramand, P., Faye, P. and Thomassier, G. (1980) Physique, Terminale C, E., Eurin-Hachette, Paris, 52-55.

[5] Bagdoo, R. (2015) Recycled Relativity. https://vixra.org/abs/1506.0125

[6] Bagdoo, R. (2016) Neutrino's Temporal Oscillations, 7, 11, 15. http://vixra.org/abs/1605.0005 https://www.academia.edu/25111027

[7] Moffat, J.W. (2009) Reinventing Gravity. Thomas Allens Publishers, Toronto, 121, $122,162,206-208$.

[8] Davies, P.C.W. and Brown, J. (1988) Superstrings. Cambridge University Press, Cambridge, 5-26-27-47.

[9] Bagdoo, R. (2008) The Pioneer Effect: A New Theory with a New Principle. http://vixra.org/abs/0812.0005 https://www.academia.edu/5535864

[10] Prigogine, I. and Stengers, I. (1992) Entre le temps et l'éternité, Champs, Flammarion, 129-133.

[11] Hawking, S.W. (1988) A Brief History of Time. Bantam Books, New York, 117, 134, 145-152. https://doi.org/10.1063/1.2811637

[12] Bagdoo, R. (2009) Arcade 2 Extragalactic Emission and Dark Matter as Seen by the Theory of Relation. http://vixra.org/abs/1302.0003 https://www.academia.edu/5538299

[13] Whyte, L.L. (1927) Archimede or the Future of Physics. Kegan Paul, Trench, Trubner and Co., Ltd., London.

[14] Cohen-Tannoudji, G. (1991) Les constantes universelles, Hachette, 112.

[15] Gamow, G. (1962) La gravitation, Payot, Paris, 136-137.

[16] Bagdoo, R. (2013) The Energy in Virtue of the Principle of Compensation. http://vixra.org/abs/1301.0180 https://www.academia.edu/5539802

[17] Whitrow, G.J. (1955) La structure de l'Univers, Gallimard. 161-169.

[18] Smolin, L. (2006) The Trouble with Physics. Houghton Mifflin, 15, 21, 22, 106, 251.

[19] Orear, J. (1967) Fundamental Physics. John Wiley and Sons, New York, 87, 100, $156,284-287$.

[20] Gribbin, J. (1991) À la poursuite du Big Bang. Champs, Flammarion, 133-134, 248, 326-328.

[21] Greenstein, G. (1983) Le Destin des étoiles. Seuil, Paris, 166.

[22] De Closet, F. (2004) Ne dites pas à Dieu ce qu'il doit faire. Édition du Seuil, 342.

[23] Atkins, K.R. (1970) Physics. John Wiley and Sons, New York, 8, 90.

[24] Ferguson, K. (1992) Stephen Hawking. Bantam Books, New York, 141-142.

[25] Trefil, J.S. (1983) The Moment of Creation. Macmillan Publishing Company, 171-175.

[26] Klein, É. (2009) Les tactiques de Chronos. Champs Sciences, 105-106. 
[27] Duquesne, M. (1974) Matière et antimatière. Presses Universitaires de France, 62.

[28] Hotson, D.L. (2002) Dirac's Equation and the Sea of Negative Energy, Part 1, Infinite Energy. Issue 43.

[29] Bagdoo, R. (2009) Link between Allais Effect and General Relativity's Residual Arc during Solar Eclipse. http://vixra.org/abs/1302.0089 https://www.academia.edu/5537473

[30] Bagdoo, R. (2013) Lunar Eclipses and Allais Effect. http://vixra.org/abs/1311.0156, https://www.academia.edu/5539813

[31] Will, C. (1989) The Renaissance of General Relativity. The New Physics, 31, 32.

[32] Davies, P. (1989) Les forces de la nature. Armand Colin, 78, 170.

[33] Stevenson, R. and Moore, R.B. (1967) Theory of Physics. W. B. Saunders Company, Philadelphia \& London, 126, 174.

[34] Jeans, J. (1937) The Mysterious Universe. University Press, Cambridge, 132-133.

[35] Adair, R.K. (1987) The Great Design. Oxford University Press, New York, 147, 209.

[36] Herbert, N. (1989) Faster than Light. Plume-Penguin Books, New York, 48-113.

[37] Silk, J. (1997) Le Big Bang. Éditions Odile Jacob, 471, 480, 568.

[38] Wilczek, F. and Devine, B. (1987) Longing for the Harmonies. W. Norton and C., New York, 43, 49, 311, 320, 363, 368.

[39] Bagdoo, R. (2011) Cosmological Inconstant, Supernovæ 1a and Decelerating Expansion. http://vixra.org/abs/1304.0169 https://www.academia.edu/5539777

[40] Cohen-Tannoudji, G. and Spiro, M. (2013) Le boson et le chapeau mexicain. Gallimard, Folio Essais, 268-275.

[41] Pagels, H.R. (1982) The Cosmic Code. Bantam New Age, New York, 5, 9, 237-243.

[42] Rousseau, P. (1950) La Conquête de la Science. Arthème Fayard, Paris, 202, 203.

[43] Cuny, H. (1971) L'aventure cosmique. Les éditeurs français réunis, 162, 166, 168, 173.

[44] Bénézé, G. (1961) Le nombre dans les sciences expérimentales. Presses Universitaires de France, 93-96.

[45] Luminet, J.-P. (2001) L’Univers chiffonné. Folio essais, 86-87, 91, 300-304, 314-316, $321,373-374,460$.

[46] Thuan, T.X. (1995) Un astrophysicien. Champs Flammarion, 19, 34, 35.

[47] Cosentino, M. (1993) Origine et destin de notre Univers par une nouvelle Cosmologie de l'atome jusqu'aux confins du Cosmos. Bonnefoy-Editeur.

[48] Tolman, R.C. (1987) Relativity, Thermodynamics and Cosmology. Dover Publication, 401-403, 412-415, 439-444, 484-487.

[49] Jolivet, R. (1965) Logique-Cosmologie. Emmanuel Vitte, Lyon, 383. 